\title{
Proceedings of the II International Symposium on Oncovirology (November, 18-20, 2004) at Sol Victória Marina Hotel, Salvador, Bahia, Brazil
}

\author{
Implementation of a Training Program on Oncovirology in Bahia \\ Carlos Brites
}

The history of Oncovirology research in Bahia began with a visit of Dr. Harrington to our site, in 1990, to propose a collaboration in HTLV research. We implemented together a wide screening for HTLV antibodies in several groups of patients, as well as in blood donors, and health care workers. In the next few years, the collaboration between University of Miami School of Medicine (UMSM) and Universidade Federal da Bahia (UFBA) became closer, and several projects were developed, with involvement of both institutions, resulting in an increasing amount of information on HTLV epidemic in Bahia. In 1999, UFBA entered the Latin American Medical Training Program, sponsored by UMSM, and co-directed by Dr. Harrington. This initiative resulted in short term fellowship at UMSM for many medical students from Bahia. Also, in 1999 was created a tissue bank for biological specimens at UFBA, as part of a subcontract with UMSM. In 2002, UFBA was formally involved in a project for development of infrastructure in cancer research, funded by American Malignancies Consortium (AMC), through UMSM. After that, Dr. Harrington and Dr. Brites applied for another grant, funded by NCI, in order to develop a program on Oncovirology research in Bahia (ICORTHA). After the grant approval, we established an ambitious training program which resulted in establishment of a research core on that field, involving Infectious Diseases specialists, hematologists, pathologists, research nurses, and administrative staff. As part of the program, an active surveillance system was created for detection and referral of patients diagnosed as viral-related cancers. A Viral Oncology Clinic was created at UFBA's Hospital (HUPES), to serve as referral center for viral malignancies. Serology for HIV and HTLV was performed in all cases of highgrade, non-Hodgkin lymphomas. In addition, a Kaposi's sarcoma clinic was established at the same site, for attending AIDS patients with that tumor.

In addition, as part of the training program, an integrated, monthly session, involving ID specialists, hematologists, and pathologists was implemented at HUPES. The session evolved from case discussion to a more complex case studies, with a very good participation of physicians and students from several fields.

Other important consequence of the program was the increasing number of samples entering the tissue bank after the starting of the protocol.

In numeric terms, in the previous 15 years a total of 119 KS cases and 35 NHL were diagnosed in the three main referral hospitals, for AIDS care, in Salvador. After the beginning of the program (18 months period), we detected a total of 73 virus-related tumors, including 13 new KS cases, 32 NHL, and 20 adult T-cell leukemia/lymphoma (ATLL).

Also as part of this initiative, we are working on detection and evaluation of Burkitt's lymphoma, to study the role of EBV in pathogenesis of disease, in collaboration with UMSM.

Bahia is a Brazilian state with very unique characteristics. In Salvador, its capital, the high prevalence of oncogenic viruses, when compared with other Brazilian cities, makes it an unique site for Oncovirology research. In addition, the already existing research infrastructure, trained personnel, and established facilities reinforce the almost ideal conditions for conducting research on that field. The finished training program strengthened the local conditions to develop different projects focusing that area. 